This is the final peer-reviewed accepted manuscript of:

Pedrosa E. et al. (2020) TIMAIRIS: Autonomous Blank Feeding for Packaging Machines. In: Caccavale F., Ott C., Winkler B., Taylor Z. (eds) Bringing Innovative Robotic Technologies from Research Labs to Industrial End-users. Springer Tracts in Advanced Robotics, vol 136. Springer, Cham.

The final published version is available online at:

https://doi.org/10.1007/978-3-030-34507-5 7

Rights / License:

The terms and conditions for the reuse of this version of the manuscript are specified in the publishing policy. For all terms of use and more information see the publisher's website.

This item was downloaded from IRIS Università di Bologna (https://cris.unibo.it/)

When citing, please refer to the published version. 


\title{
TIMAIRIS: Autonomous Blank Feeding for Packaging Machines
}

\author{
Eurico Pedrosa, Gi Hyun Lim, Filipe Amaral, Artur Pereira, \\ Bernardo Cunha, José Luís Azevedo, Paulo Dias, Ricardo Dias, \\ Luís Paulo Reis, Nima Shafii, Andrea Tudico, Claudio Mazzotti, \\ Marco Carricato, Simone Badini, Dario Rea and Nuno Lau
}

Abstract Current packaging machine vendors do not provide any automated mech
anism for blank feeding and the state of the art is to have a human operator dedicate
to feed the blank piles to the packaging machine. This is a tedious, repetitive
E. Pedrosa · F. Amaral · A. Pereira P. Cunha J. L. Azevedo · P. Dias · R. Dias
Institute of Electronics and Informatics Engineering of Aveiro, University of Aveiro, Aveiro,
Portugal
e-mail: efp@ua.pt
F. Amaral
e-mail: f.amaral@ua.pt

A. Pereira

e-mail: artur@ua.pt

B. Cunha

e-mail: mbc@det.ua.pt

J. L. Azevedo

e-mail: jla@ua.pt

P. Dias

e-mail: paulo.dias@ua.pt

R. Dias

e-mail: ricdays@gmail.com

G. H. Lim

School of Computer Science, University of Manchester, Manchester, UK

e-mail: hhmetal@gmail.com

L. P. Reis

University of Porto, Porto, Portugal

e-mail: lpreis@fe.up.pt

N. Shafii

NVIDIA, Munich, Germany

e-mail: nima.shafii@gmail.com
A. Tudico
University of Bologna, Bologna, Italy
e-mail: tudico.andrea@gmail.com 
tiring task. This also results in problems with unintentional errors, such as using the wrong pile of blanks. An alternative solution is the use of a fixed robotic arm surrounded by a protective cage. However, this solution is restricted to a single packaging machine, a unique type of blank shapes and does not cooperate with humans. TIMAIRIS is a joint effort between IMA S.p.A., Italy, (IMA) and the Universidade de Aveiro, Portugal, (UAVR), promoted by the European Robotics Challenges (EuRoC) project. Together, we propose a system based on a mobile manipulator for flexible, autonomous and collaborative blank feeding of packaging machines on industrial shop floor. The system provides a software architecture that allows a mobile robot to take high level decisions on how the task should be executed, which can depend on variables such as the number of packaging machines to feed and the rate of blank consumption at each one. Through a computer vision system, blanks of different shapes and sizes are correctly identified for adequate manipulation. The manipulation of the piles of blanks is performed using a single arm using compliant modes of operation to increase manipulation safety and robustness. Additionally, it has a safe navigation system that allows the robot to be integrated in an industrial environment where humans are present. Finally, it provides an enhanced multimodal interaction between human and robot that can be adapted to the environment and operator characteristics to make communication intuitive, redundant and safe.

Keywords Mobile manipulation · Planning - Industrial perception · Logistics

\subsection{Introduction}

The task of blank feeding a packaging machine, a known industrial logistics problem, is nowadays mostly performed by a human operator (see Fig. 7.1). It is a tedious, repetitive and tiring task for a human where occasional errors are unavoidable. On an effort to reallocate the human operator to more interesting tasks and decrease

C. Mazzotti $\cdot$ M. Carricato

University of Bologna - IMA S.p.A., Bologna, Italy

e-mail: MazzottiC@ima.it

M. Carricato

e-mail: marco.carricato@unibo.it

S. Badini · D. Rea

IMA S.p.A., Bologna, Italy

e-mail: BadiniS@ima.it

D. Rea

e-mail: ReaD@ima.it

N. Lau $(\varangle)$

Instituto de Engenharia Electrónica e Informática de Aveiro, Universidade de Aveiro, Campus Universitário de Santiago, 3810-193 Aveiro, Portugal

e-mail: nunolau@ua.pt 

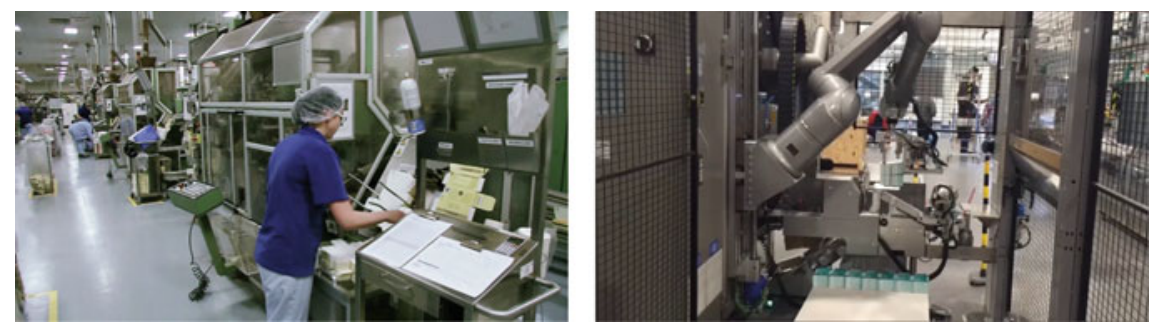

Fig. 7.1 State-of-the-art in the packaging industry. Blanks are feed manually (left image) or by a robotic arm in a cage (right image)

production downtimes due to mishandling of blanks, a fixed robotic arm surrounded by a protective cage was implemented by the industry as an alternative solution for blank feeding, as shown in Fig. 7.1. However, this solution is bounded to a single packaging machine, restricted to a unique type of blank shape and it is incapable to cooperate with humans (i.e. the robot and humans do not share the same physical space). This lack of flexibility is one of the obstacles that the INDUSTRY 4.0 initiative $[1,2]$ wants to remove from the industrial shop floor.

Research and implementation of flexible and autonomous solutions for logistic problems, such as blank feeding, can be accelerated by strengthening collaboration between the industry and the academia. This was the objective of the European Robotics Challenges (EuRoC) european project. By proposing a research project based on a robotic competition, it drives innovation in robotics applied to industrial manufacturing and logistics problems [3]. In summary, an industrial partner provides a use case and the academic partner offers a solution. This requires the exchange of knowledge between the partners to achieve a common goal, an innovative and productive solution based on flexible autonomous robots. The competition component of the project also has an important role; not only it promotes healthy competition between different partnerships but also offers additional funding for the most promising solutions. It was in the context of EuRoC that TIMARIS team was created. This partnership is a joint effort of the Intelligent Robotics and Systems (IRIS) group from Aveiro University (UAVR) in Portugal and IMA S.p.A. (IMA) from Italy. Together we proposed to develop a system for flexible, autonomous and collaborative blank feeding of packaging machines on industrial shop floor.

Our approach meets the requirements of a modern industry by rationalising the exploitation of raw materials, energy and repetitive human labour. The employment of robotised systems also increases the safety of workers' activity, since risky tasks can now be accomplished by automatised manipulators. This proposal intends to develop and transfer technologies from academia to industry to enable industrial robots with an architecture that allows them to: take high level decisions on how should the task should be executed (planning, replanning and optimization); integrate the robot in an industrial environment where humans are also present and moving (safe navigation; collaboration) [4]; be able to identify, locate and manipulate several types of blanks in an unstructured environment (computer vision, manipulation); benefit from the 
use of an enhanced multimodal human-robot interaction that can be adapted to the environment and operator characteristics, making it more intuitive, redundant and safe (communication).

\subsection{Use Case Description and Motivation}

Even in modern automatic machines, the final-product boxes are provided by the manual feeding of flat or pre-glued blanks. Loading blanks should be performed by robotised systems. This implies several advantages: it frees up the operator from the fixed rhythm, ensures an overall improved machine energy efficiency and leads to a more sustainable packaging process. Although the use of robots for manufacturing and logistics is common in the industry for several decades, the environment where these robots operate is, in general, heavily restricted, as is the case for most applications of robotic arms in industry, or the robots must follow a predefined set of paths that severely restrict their use in environments where humans are also moving. Interaction between the robot and humans is also quite limited and mostly performed by reprogramming the robot or by simple button-based input tools and LCD screens.

The TIMAIRIS blank feeding system intends to develop and transfer technologies from academia to industry to enable industrial robots with an architecture that: allows them to take high level decisions on how should the task be executed (planning, replanning and optimization); integrates the robot in an industrial environment where humans are also present and moving (safe navigation); is able to identify, position and manipulate several types of blanks in an unstructured environment (computer vision, manipulation); has an enhanced multimodal interaction between human and robot that can be adapted to the environment and operator characteristics to make it more intuitive, redundant and safe (communication). The end-goal of our team, within the EuRoC project (in which we participated in Challenge 2-Shop floor logistics and manipulation), was to develop a pilot of the "Autonomous Blank Feeding for Packaging Machines" task on an industrial shop-floor. But to reach the final goal, there was a set of preceding use-cases that had to be solved and evaluated as part of the EuRoC competition workflow. This workflow was an interesting innovation introduced by the EuRoC project. The use-cases that were developed before the Field Tests were the Benchmarking, Freestyle and Showcase introduced in the next sections.

The robotic platform used to address the use-cases, including the Field Tests "Autonomous Blank Feeding for Packaging Machines", was provided by the EuRoC Challenge host (German Aerospace Center: DLR-Deutsches Zentrum für Luft). It was a KUKA omnirob with a mounted Light-Weight-Robot (LBR) equipped with a two jaw-gripper and a stereo camera, and a pan-and-tilt unit also equipped with a stereo camera. Access to the cameras, the LBR and mobile platform was provided by a Robotic Operating System [5] (ROS) wrapper, hence all developments through the project were made using the ROS environment. This platform was common to all teams participating in EuRoC Challenge 2. 


\subsubsection{Benchmarking}

In the Benchmarking use-case, all Challenge 2 participating teams had to solve the same two tasks defined by the challenge host. The first task was a logistics problem that required several small load carriers (SLC), which were distributed in the room, to be placed within a fixed target area. The objective was to recognize the SLCs using computer vision and use arm manipulation to pick them and place them in the target area as fast as possible without dropping the production materials inside the SLCs. The second task was a product pre-assembly problem that required the use of a single anthropomorphic robotic arm to assemble several bolts with the proper washers and nuts with the help of a mounting fixture. The objective was to put the washers in the bolts and screw their nuts as fast as possible. The task of pre-assembling a single bolt requires several steps: identify the mounting fixture position: recognize the bolt, grab it and place it in the mounting fixture; identify a washer, grab it and place it in the nut; and finally find a nut, grab it and screw it in the bolt until the bolt's top is not occluded by the nut.

Without neglecting the competition part of this use-case, the Benchmarking was used by TIMAIRIS to learn on how to use the provided robotic platform and develop a software architecture to be used through the remaining use-cases, including the Field Tests. The end result is a skill-based anytime-agent architecture for logistics and manipulation tasks $[6,7]$ that was successfully used in all subsequent use-cases.

\subsubsection{Freestyle}

The Freestyle use-case was intended to develop and demonstrate technologies for collaborative problem solving between humans and robots using flexible multimodal communication. Based on the previous objectives, EuRoC teams were free to define the specification of the Freestyle task based on their expertise and on having in mind the final objective of their use case. The specification of the Freestyle task was performed in the submitted proposal to be admitted to EuRoC Stage II (benchmarking, freestyle and showcase). The TIMAIRIS task consisted in assembling a puzzle using a set of pentominoes pieces (formed by joining 5 cubes, face by face, on a single level). Assembling is assumed to be done cooperatively by a human and a robot. The highlights of TIMAIRIS Freestyle use-case are summarized in the following list:

- Perception: fusion of RGB/Depth information is used to detect the pose of colored and uncolored pentominoes, the pose of the fixture where the puzzle is assembled and also the puzzle state.

- Navigation/manipulation: planning and execution for safety/compliance in a human populated set. The robot has to pick pentominoes from the picking station, transport them, and deliver them in several different ways to the human, including placing it in the human hand, or assemble the pentaminoe in its final 
position in the puzzle. Movements must be coordinated and safe, as the human also picks and transports pentominoes and assembles the puzzle.

- Planning and replanning: the proposed game can be very challenging for a human player. The high computational power of the robot allows the implementation of effective planning algorithms for solving the puzzle that can be interactively used to assist the human in finding the puzzle solution.

- Multimodal interaction: having several communication modalities that can be combined together allows for robot adaptation to different users and environments. During the free-style the following modalities were used: gestures; voice; graphical user interface (images and keyboard/mouse).

The Freestyle use-case was deliberately decided to be significantly different from the Showcase and Field Tests use-cases, but it demonstrates common functionalities with the showcase and field tests such as perception, navigation/manipulation, planning, multimodal interaction and cooperation.

\subsubsection{Showcase}

The Showcase use-case was composed of a simplified version of the complete autonomous blank feeding problem, that included a prototype of a blank magazine, the mechanism that feeds the blanks into the packaging machine, and two pallets filled in with actual blank piles used in production. The task consisted in recognizing the blank piles on two different pallets, devise a plan for picking them, and transport and place them in the blank magazine in a smooth way so it does not get jammed. The workspace is assumed to be shared by humans and the robot. The main issues addressed by this use-case are the following:

- Perception: Fusion of RGB/Depth information is used to detect the pose of the pallets, blank piles and also the pose of the blank magazine; multiple laser rangefinders are merged to detect and track humans in the workspace. Compliance aplied to the manipulator movements is used to increase safety and to detect abnormal situations that result in non valid arm positions and also allowing the trigger of recovery procedures.

- Navigation/manipulation: Using a single arm, the robot has to pick blank piles from two pallets that require navigation planning and arm motion planning for picking and placing the blank piles. It should be noted that blank piles are composed of several distinct objects and have no obvious grasping position when laying on top of the pallets. For safety concern, the presence of a human in the vicinities of the robot triggers a generalized motion stall.

- Planning: Due to the blank piles arrangement on the pallets, there are piles that need to be picked first or, otherwise, manipulation will not be possible for all cases. Hence, that requires the generation of a plan establishing the picking order. Task execution is monitored while being executed and if deviations are detected 
recovery plans are triggered. Detection of situations like "need for blanks" from the magazine is also included.

- Multimodal interaction: Having several communication modalities that can be combined together allows for robot adaptation to different users and environments. During the Showcase the following modalities were used: gestures; text-to-speech; visual feedback on a tablet; graphical user interface (images and keyboard/mouse). Humans near the robot are tracked using laser scanners and, when within the interaction distance, the pan-tilt camera keeps its attention on the closest human to recognize possible gesture commands.

The showcase scenario has been considered to solve pending problems for autonomous packaging. In automatic packaging, blank piles have to be manually fed by human operators, who may occasionally refrain from collecting blank piles from more distant pallets.

\subsubsection{Field Tests}

Since IMA produces and delivers packaging machines for many kinds of products such as pharmaceuticals, cosmetics, tea, coffee, dairy and food, and those products continue to rapidly evolve, human operators and autonomous robots are required to adapt to changing environments in a short period of time. This environment is less structured and demands higher flexibility than large-scale or mass-production industries. The Field Tests use-case aimed to demonstrate a solution for the blank feeding problem in the shop floor environment and using actual functional packaging machines, including sharing the space with human operators. There is a high motivation for automating labour intensive problems such as loading blanks. This is an essential operation, but one that involves little added value. The main ideas for enriching the final task compared with the Showcase (that was a simplified version from this final use-case) are mainly the following:

- Blank identification and pose estimation: capability for identifying several types of blanks as well as their orientation within the pallet, which may change from pile to pile;

- Multiple machine feeding: capability to feed more than one machine with the appropriate blank type;

- Planning: prediction and intelligent scheduling of blank feeding operations;

- Pile blank grasping: gripper with capabilities for grasping distinct types of blanks, the robotic solution also increases the human picking capability by allowing piles as high as around $15 \mathrm{~cm}$ and weighting around $6 \mathrm{~kg}$ to be pick in a single movement;

- Safety management: navigation and manipulation in an environment that is shared with human operators;

- Advanced communication/interaction: capability to interact with human operators through a multimodal interface. 
The Field Tests setup required two packaging machines, and a set of pallets with at least two types of blanks (available at the end user). The position of the pallets and machines was similar to those found in real deployed shop floors. The orientation and position of the blanks on the starting pallet may vary according to the printing layout. Equipment also includes the KUKA platform and a new blank gripper, different from the one used in the Showcase. During these operations, the interaction between human, machine and robot clearly has to take place in conditions of safety, considering that the operator's job is to oversee the functioning of the machine and to intervene if anything goes wrong. The multimodal and bi-directional interaction between humans and the robot allowed an effective human machine interface (HMI) for robot-operator communication. Blanks are supplied on pallets, using flat blanks that are already creased and die cut. The pallets can be raised from the floor level to allow the manipulator mounted on the KUKA omniRob to grasp any block of blanks within the pile.

\subsection{Activities and Results}

\subsubsection{Benchmarking}

The goal of our team was to solve the Benchmarking use-case, proposed by EuRoC, with high precision, accuracy, robustness and as fast as possible. Time is a key factor while solving the tasks at hand. Not only it is an important evaluation metric, but for industrial application it is a matter of productivity.

\subsubsection{Skill-Base Anytime Agent Architecture}

The developed software architecture is divided in several functional components, as shown in Fig. 7.2. The Perception modules are responsible for collecting sensory data and process them to extract the relevant information used in high-level modules such as the Solver. A manipulation/navigation Skill is the capability of doing a particular action/task, such as, picking and placing an object or moving the manipulator's endeffector to a desired pose. Perception modules and Skills obtain and send sensorymotor data via the Sensor and Effector interfaces, respectively. It is the responsibility of the Solver to take decisions on how to solve the current task based on the available sensory information and available Skills. The Action Planner provides a sequence of skills in order to manipulate the current object of interest. Finally, the Order Graph represents the objects in the environment and the order in which they are manipulated. The order is restricted by a directed acyclic graph that represent a dependency between objects in terms of order of manipulation. For example, leaf object have to be handled before its parents, a situation that is ubiquitous in assembly problems (e.g. bolt assembly, puzzle assembly). 
Fig. 7.2 Overview of the skill-based agent architecture

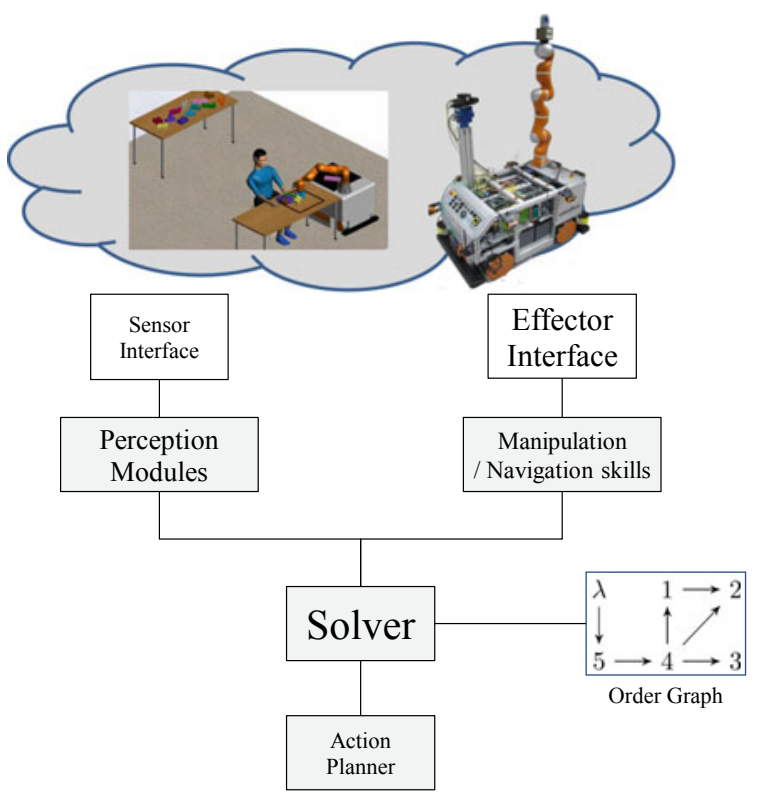

\subsubsection{Production Logistics Task}

The objective of this task is to find and pick all SLCs present in the room and place them in the designated area. Their approximate locations are known, i.e. on the table or in the shelf, but their exact location is not known. To guarantee that all SLCs are eventually detected the task starts with several search poses that cover the complete known area. Once a SLC is sighted its pose should be detected. Once the pose is obtained the agent decides how to pick and where to place it.

The SLC detection has two stages: first, color segmentation is used to candidate image regions that may contain an SLC; second, a 3D point cloud for each region is created and matched against a 3D template of the SLC to find its position and orientation (i.e. its pose). An example of SLC detection is shown in Fig. 7.3. The color segmentation, in HSV color space, takes advantage of the color homogeneity of the SLC and good contrast with the environment to reduce the search space. The output of this segmentation is a set of blobs that represent SLC candidates. However, blobs must have a minimum area to be valid. Overlapping SLCs in the image is a possibility, but that is not a concern as disambiguation is deferred to the next stage.

After color segmentation, the resulting blobs are used as masks to generate a pointcloud for each candidate. Let $P$ be a pointcloud and $p_{i}$ the set of points of $P$. To reduce the computational complexity of processing the pointcloud with a high number of points, $P$ is decimated by applying a voxelization filter. Then, to remove possible outliers a statistical outlier removal is also used [8]. To handle the existence of overlapping SLCs, the point cloud is divided into clusters using an Euclidean clustering method [9]. The cluster with the highest number of points is assigned to 

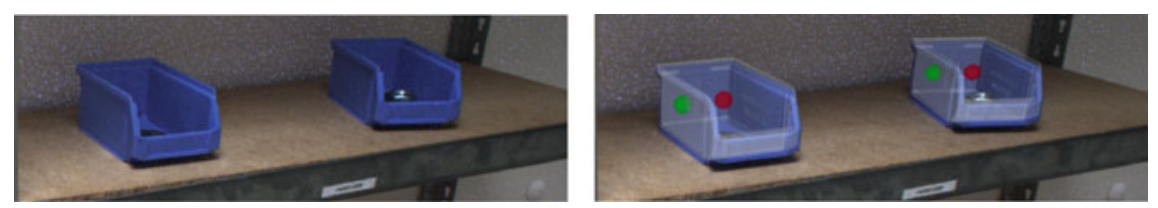

Fig. 7.3 Example detection of two SLCs in a shelf. The left image is what is perceived by the stereo camera (image plus depth). The right image contains a superimposed 3D model of the SLC for each detection, including their center (red dot) and possible pick point (green dot)

$P$ and the remaining points are discarded. Due to our agent architecture, discarded SLCs will be detected in the next cycle. The position of the SLC is approximated by the pointcloud centroid $c$ given by $c=\frac{1}{n} \sum_{i=1}^{n} p_{i}$ while its orientation is initially provided by the Principal Components Analysis (PCA) of the projection of $\mathrm{P}$ in the XOY plane [10]. The view of the SLC may provide a partial pointcloud that skews the centroid from the real center and the orientation of the PCA has an ambiguity of $\pi$ radians. Therefore, the final pose of the SLC is calculated by matching $P$ against a 3D template of the SLC that has its centroid in the origin and orientation aligned with the $\mathrm{X}$ axis. Before matching, the pointcloud $P$ is transformed to its origin (i.e. its inverse pose applied to itself) so the transformation that results from the matching is the correction of the initial pose calculation. Because the orientation given by PCA is ambiguous, both orientations are used for matching. The pose with the lowest error is then selected as the final pose. The algorithm used for matching is the extension of the scan matching algorithm proposed by Pedrosa et al. [11] to three dimensions.

\subsubsection{Product Assembly Task}

The purpose of this task is to pre-assemble a set of bolts, nuts and washers using a single anthropomorphic robotic arm mounted on a mobile robot. To help the assembly, two fixtures are provided. The first hold the washers in (almost) upright position to help manipulation. The second has a well with the shape of the bolt's head, shallow enough to hold the bolt in place while the nut is being put in place. The bolts and nuts are in the vicinities of the fixtures (see Fig. 7.4). The approximate location of the assembly pieces and fixtures are known, but not their exact position.

The TIMAIRIS solution to this problem first moves the robot from the center of the room (mandatory robot location for starting this task) to a location near the table where the bolts, nuts, washers and fixtures are. Then, using the camera attached to the end-effector, the scene is observed from a point of view that provides a complete view of all necessary elements for the task without further need to change the position of the mobile platform. By keeping the mobile platform fixed, the relative position of objects to the manipulator is maintained and we can take advantage of this assumption to reduce the number of observations with the camera.

To execute the task the agent has to detect the bolt, pick it and place it in the bolt fixture, then detect the washer, pick it and place it in the bolt, and finally it has to 


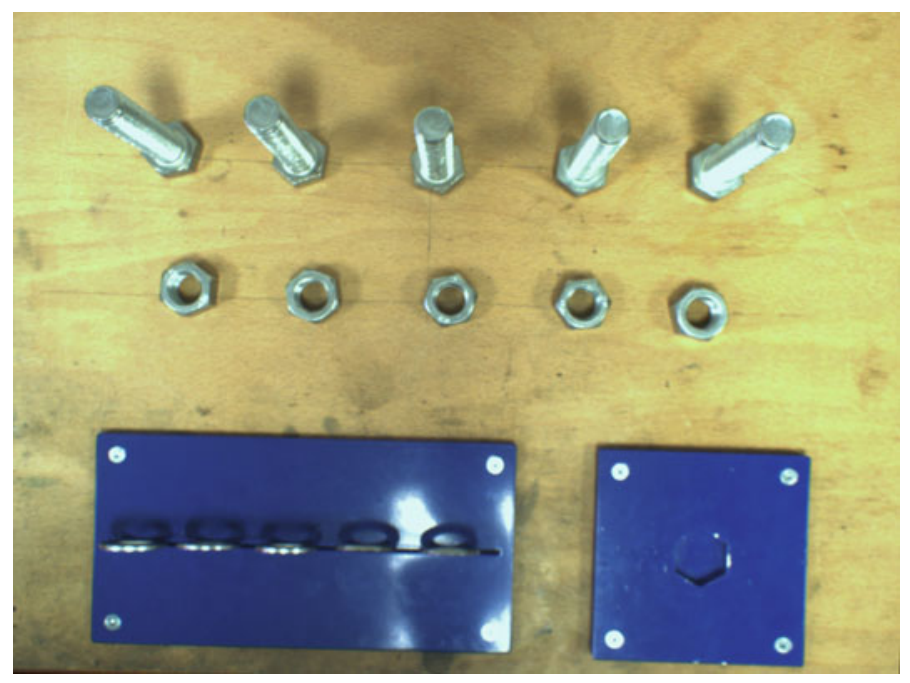

Fig. 7.4 Example of an initial setup for the product assembly task

detect a nut, pick it and screw it in the bolt. To calculate the positions of this objects we use the pinhole model instead of the pointcloud provided by the stereo camera. We can follow this strategy because the dimensions of all elements in the task are known and the distance from the camera to the working table is known in advance. By avoiding the creation of depth information we reduce the computational complexity and speedup the detection operation.

\section{Fixture Detection}

The detection of the fixtures, although executed only once, is an important step. Because the mobile robot has to navigate to the working area at the beginning of the task localization, some uncertainty in its absolute position is inevitable. However, the assembly area is well defined, therefore we can use the location of the fixtures to define the reference frame for the remaining elements. The detection of the fixtures is performed using color segmentation on the HSV color space. An initial, rough observation, is performed from the observation point of view. The resulting blobs are then used to calculate the rotated rectangle that best encloses each individual blob. The detection from the observation point does not provide enough accuracy for using the fixtures, therefore, using the information from the rotated rectangles, we approximate the camera to each fixture and repeat the detection individually for each fixture. The localization of the fixture that contains the washers is used in the detection of the washers and the center of the rotated rectangle that derives from the bolt's fixture coincides with the screwing well. 


\section{Bolt, Nut and Washers Detection}

The detection algorithm for the bolts and nuts is the same. From the top view, close to the image center, these elements share a similar geometry. The detection of a bolt/nut starts by performing color segmentation (in HSV color space). Only the resulting blob closer to the image center and in the vicinity of the bolts virtual line is considered-both nuts and bolts are placed in the neighborhood of a fixed distance from the fixtures, hence the virtual line. By performing shape analysis we can obtain the vertices of its convex shape (i.e. ideally an hexagon) and try to find two consecutive edges that match the hexagonal shape in length and angle (see Fig. 7.5). These edges are then used to calculate the center of the bolt/nut and its orientation. The orientation is only needed for the nuts so that they can be picked by their edges.

The described approach assumes that the object to be detected is near the image center. This is achieved by generating a hint list for bolts and nuts, from the initial observation view using color segmentation, that provides an approximation of their position. When it is time to detect a bolt/nut, the hint is used to approximate the camera to the object of interest. The washer detection uses a different strategy. Instead of color segmentation an intensity filter is used to obtain the pixels with higher intensity (see Fig. 7.6). The resulting blobs are validated by their size. The centers of theses blobs are then used to calculate the position of the washers.
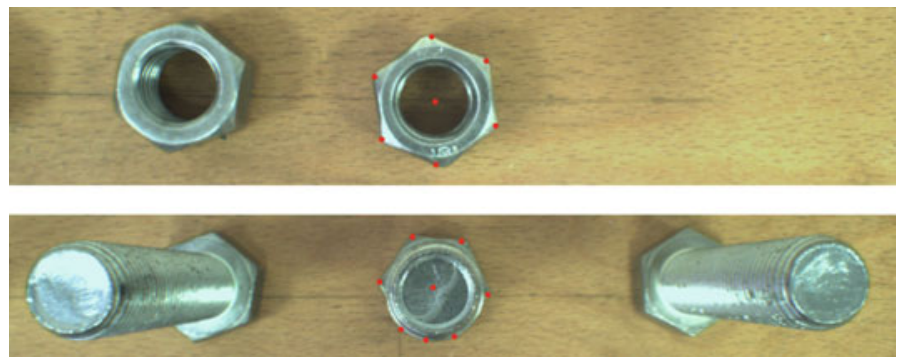

Fig. 7.5 Example of nut and bolt detection. The top image contains the detection of a nut and the bottom image contains the detection of a bolt. The red dots identify the object center and the shape vertices
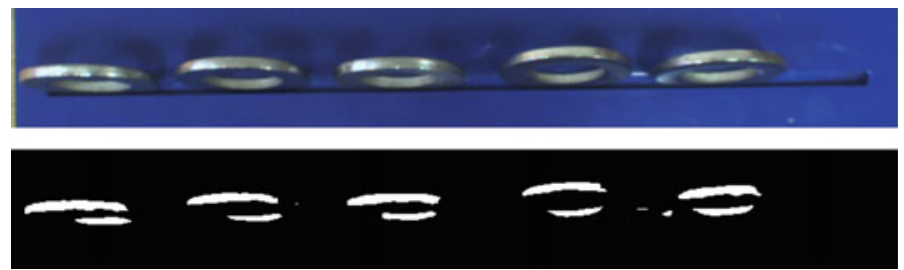

Fig. 7.6 Washer detection in the fixture. The top image is what is perceived by the camera. The bottom image is the result of the intensity filter 


\subsubsection{Motion and Manipulation}

Extracting the information from the environment is only part of the task. Physical objects have to be moved in order to fulfill the task. Time is a critical metric in industrial applications. During development velocities were kept to a minimum to prevent possible physical harm and velocities were only increased when the combination of perception + manipulation proved to be reliable. In the Production Logistics task the base of the mobile platform was used as a buffer to store SLCs so that the number of motions of the mobile platform was optimized. In the Product Assembly, manipulation with compliance was fundamental. Using compliance, it was possible to put the bolt in the screwing fixture without visual confirmation by pushing and rotating the bolt until it fell inside the fixture well. Additionally, by using compliance, we were able to produce a soft vibration effect to the bolt while putting the washer in place that helped with possible stuck situations.

\subsubsection{Results}

The developed software architecture provided a solid base from which different industrial applications can be developed. Beside the two tasks of the Benchmarking use-case, all the remaining tasks of the EuRoC project were solved with the proposed architecture. It provided a high reusability of several components for different applications, most notably the motion and manipulation Skills. The perception modules that we developed for the Benchmarking use-case proved to be accurate, precise and had a high level of repeatability. The Production Logistics task was solved in 3 m:4 $\mathrm{s}$ while the Product Assembly task was solved in $6 \mathrm{~m}: 15 \mathrm{~s}$. For both tasks our Team, TIMAIRIS, obtained the fastest results among all EuRoC Challenge 2 teams with an advantage of $1 \mathrm{~m}: 58 \mathrm{~s}$ in the Logistics task and $8 \mathrm{~m}: 45 \mathrm{~s}$ in the Assembly task to the closest competitor.

\subsubsection{Freestyle}

The Freestyle use-case was performed using an area of around $16 \mathrm{~m}^{2}(4 \mathrm{~m} \times 4 \mathrm{~m})$. Setup consisted of a picking station, where the pentominoes initially lie, an assembling station, where the puzzle is assembled (the picking and assembling stations were not simultaneously accessible by the robot from the same spot), a set of 12 pentominoes (one of each different kind), a human player and the KUKA mobile platform (see Fig. 7.7). A depth sensor (e.g. Kinect) was used to recognize the gesture commands and two external monitors were used to convey feedback to the human/evaluators. The assembling station included a clearly defined area where the fixture that defines the position of the puzzle was placed by someone from the EuRoC C2 host. The picking station also had a clearly defined area in which the pentominoes were placed randomly by someone from the EuRoC C2 host. In those cases 


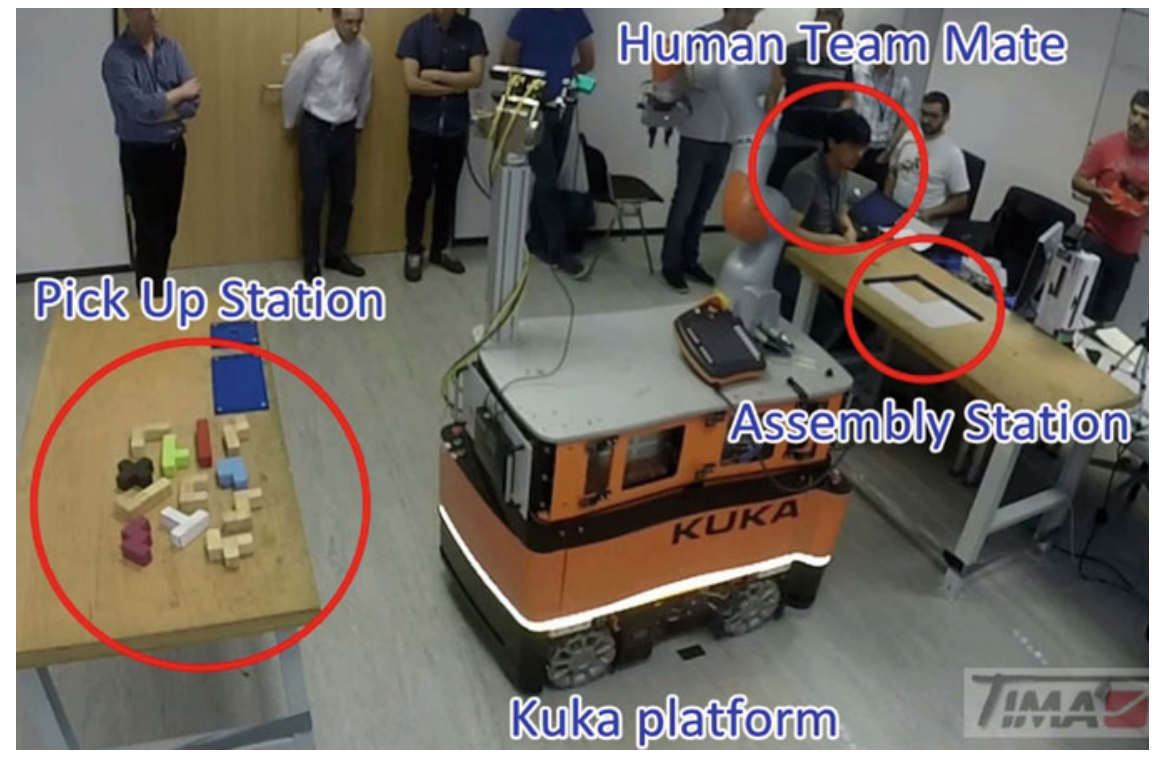

Fig. 7.7 Freestyle environment layout

in which not all pentominoes were used (challenges 2 and 3, see below), half of the pentominoes were chosen by a host member and the other half by TIMAIRIS members.

\subsubsection{Pentominoes Puzzle Assembly}

The task of assembling the puzzle is shared by a human operator and the robot. It is the responsibility of the robot (or robotic agent) to recognize the available pieces, devise a plan in order to assemble the puzzle and transmit that information to the operator (if requested). During assembly, the robot may have to replan, i.e. calculate a new puzzle solution, due to actions performed by the operator. Any action performed by the robot is always the result of a request by the operator.

\section{Pentominoes Detection}

The detection of the pentominoes is achieved by extracting shape information from an image combined with depth information (represented as a point cloud). Shape information is used because the pentominoes do not have unique colors and simpler approaches such as color segmentation failed to uniquely identify each piece. The first step of pentominoes detection is to remove from the point cloud the points that belong to the picking station tabletop. RANSAC is used to detect he tabletop points and all 

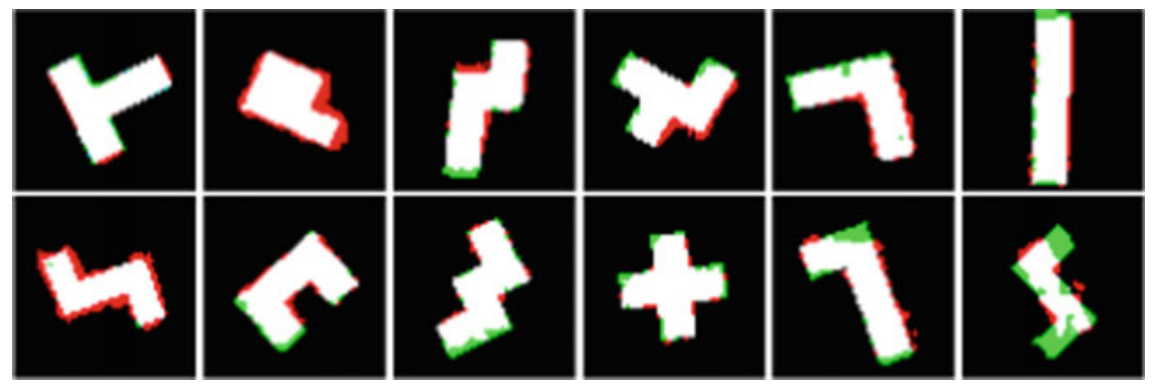

Fig. 7.8 Example of pentominoes detection with template matching. Each image corresponds to a detected pentomino that best matches the cluster of projected points

points below the tabletop (plus tolerance) and higher than tabletop plus pentomino height (plus tolerance) are removed from the pointcloud. The remaining points are assumed to be part of the top surface of the pentominoes. Those points are projected to the $\mathrm{XY}$ plane and then an Euclidean Clustering is used to extract individual pieces into clusters. Each of the clusters is then checked against all scaled templates of pentomino shapes in every rotation. Two metrics are extracted: percentage (\%) of cluster area covered by the pentomino template, and \% of template area covered by the cluster. The templates used for the 2 metrics are slightly different. The first metric uses pentomino templates that are slightly larger than the ideal pentomino template, so that small deviations of the cluster from the template do not decrease this metric, The second metric uses templates that are slightly smaller than the ideal template, to allow small imperfections on the border of the cluster. If the thresholds for both metrics are surpassed, then the pentomino position and orientation are discovered. This procedure allowed for a robust perception (e.g. Fig. 7.8).

\section{Planning}

Considering the available pentominoes, an assembly plan is computed considering the constraint that each piece has to be placed on the table and then pushed into its position. This constraint is what allowed us to also use the robotic arm (not just the operator) to assemble the puzzle. After each piece is placed in the puzzle the robot re-evaluates its current plan by comparing the current state of the puzzle with its internal representation. If both states do not match, it replans the puzzle assembly. The replanning situation happens when the operator does not follow the assembly instructions provided by the robot. An example of a plan is depicted in Fig. 7.9. 

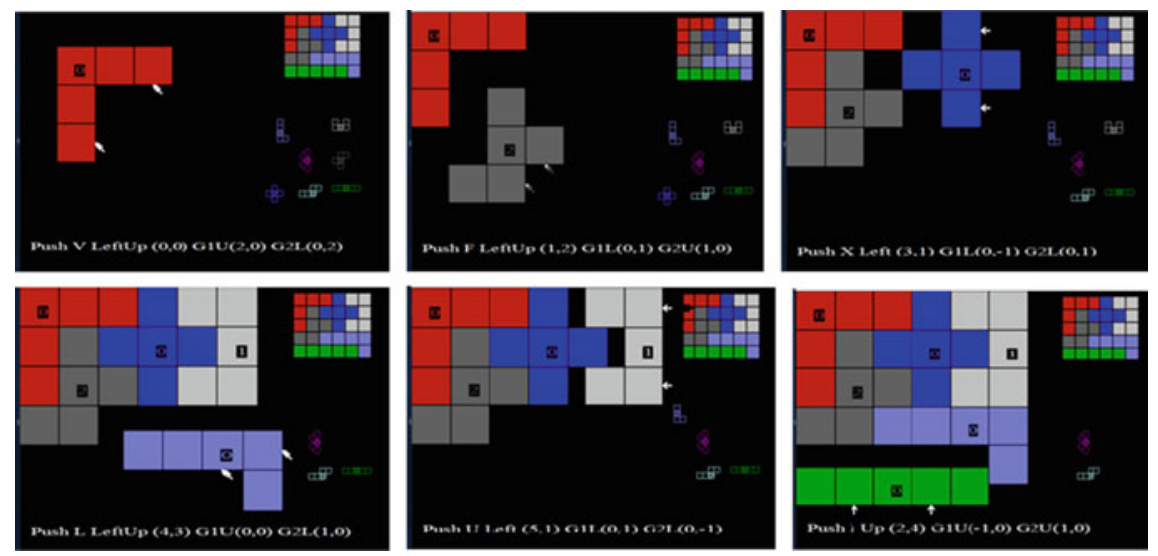

Fig. 7.9 Example of a puzzle assembly execution plan. Given the available pentominoes the plan provides the picking order and the push points and direction

\subsubsection{Human-Robot Interaction Through Gestures}

For the Freestyle use-case we introduced a Human-Robot Interaction (HRI) system to allow a human to communicate with the robotic agent through gestures [12]. This HRI was used to solve the pentominoes assembly in collaboration with the robot. A gesture language was used instead of speech to allow its usage in noisy environments, such as industrial shop floors [13]. This language is composed by eight one-hand gestures that form the base symbols, as shown in Fig. 7.10. Similar to human language, a limited number of symbols can be used to compose an unlimited number of sentences. For this use case we defined an interaction graph (see Fig. 7.11) that generates 24 sentences (or commands) using the available gestures. A sentences is composed by concatenating the gestures as sentences.

The gesture recognition is achieved with the combination of convolutional neural networks (CNN) and a contour based hand feature. The HRI system needs to construct a sentence from the continuous gesture recognition results. A confidence-based method is applied to concatenate a sequence of symbols in a robust manner [14]. An interval-counter for each gesture is defined on the basis of confidence law of inertia, where a recognized symbol is assumed to persist unless there is a confidence to believe otherwise. Additionally, applying a spelling correction from the word corpus [15], the HRI system can infer the complete command set with increased robustness. A complete description of the gestures representation and recognition can be found in the work presented by Lim et al. [12]. 


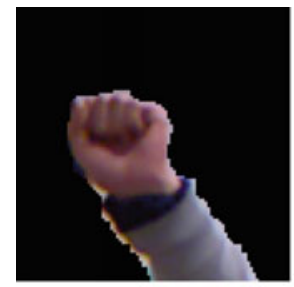

0

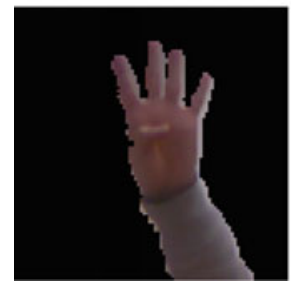

4

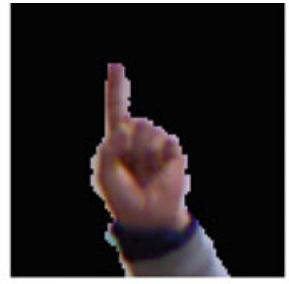

1

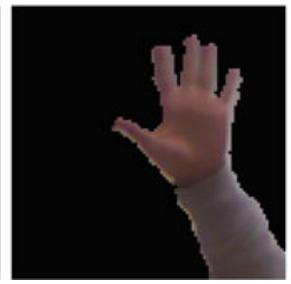

5

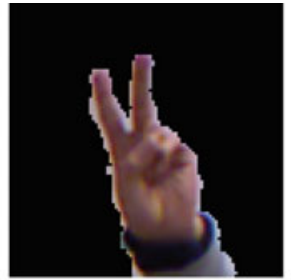

2

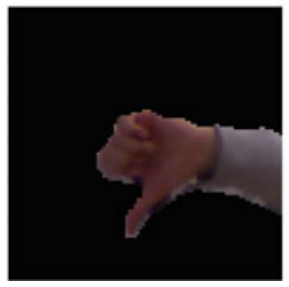

6

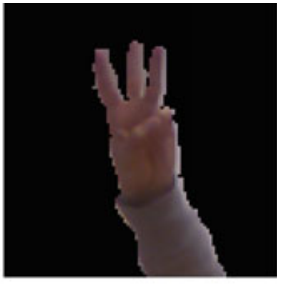

3

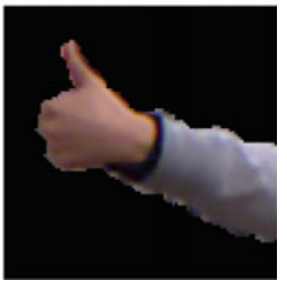

7

Fig. 7.10 The eight one-hand gestures and their corresponding IDs

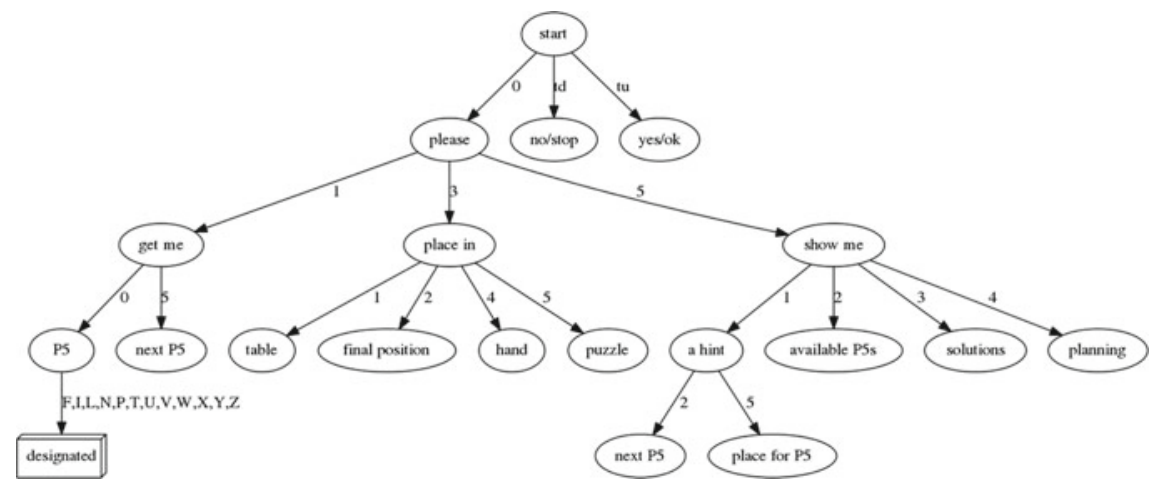

Fig. 7.11 Interaction graph that defines the communication with the robotic agent. Each leaf in the interaction graph correspond to an action performed by the robotic agent

\subsubsection{Scenarios}

The Freestyle use-case execution consisted of three different scenarios with increasing complexity. Here is their description:

Scenario 1 The human operator stays seated at the assembly station; he asks the robot to pick the next pentominoes for a possible solution; the robot gets it from the picking station; then the robot shows the final position of the pentominoes; the robot delivers the pentominoes; the human places the pentaminoe in the designated place; the process is repeated until the puzzle is finished; 12 pentominoes are available at picking station; the puzzle can consist of 5 or 6 pentominoes; 
Scenario 2 The difference here is that the human always asks the robot to pick a specified pentaminoe; whenever needed, the human can ask the robot for a hint regarding the next move, which he can choose to follow or not; giving a hint implies that the robot must perceive the current status of the assembly process and plan or replan a solution accordingly; the robot can provide the number of solutions and the complete assembly plan; 8 pentominoes are available at picking station; puzzle uses 5 of the 8 pentominoes;

Scenario 3 In the last scenario, symmetrical cooperation is introduced. The initiative regarding the next move is given alternately to the robot and the human. Considering the characteristics of the task, both the robot and the human may ask the partner to fetch a pentaminoe or to assemble a given pentominoes in the puzzle; the robot can provide the number of solutions and the complete assembly plan; 8 pentominoes are available at the picking station; the puzzle to be assembled can consist of 5 or 6 pentominoes.

Each scenario had several metrics that were used to evaluate our performance as EuRoC challengers. Those metrics were focused on capabilities such as properly identifying the pentominoes and recognizing the operator gestures.

\subsubsection{Results}

The final outcome of the Freestyle use-case was a scenario where a human operator and a mobile robot successfully worked together to assemble a pentomino puzzle, using only gestures to communicate. During evaluation, most target metrics were reached (or surpassed) that provided us a second place in the evaluation (out of five).

The challenges provided by this use-case were centred around the development of pentomino detection, puzzle assembly planning and HRI system. The integration of these technologies into a working system was simplified by our software architecture due to high reusability of already developed components for the Benchmark use-case.

The Freestyle use-case is significantly different from the showcase and field test use-cases, but demonstrates common functionalities such as perception, manipulation, planning, multimodal interaction and cooperation. In fact, detecting piles of blanks, picking, transporting and placing them in the magazine (summary of our showcase/field tests experiment) is a task that has the same structure as detecting, picking, transporting and placing pentominoes.

\subsubsection{Showcase}

The Showcase use-case was performed in an environment that, although not located in an industrial plant, includes the most important elements of the real environment where the task was supposed be executed as a final product. It uses real pallets with real piles of blanks and a prototype of the blank feeding mechanism of a packaging 
machine that includes all relevant features for the task to be performed. In the industrial setting, the distance between the blank feeding mechanism and the pallets may be larger, there may be several packaging machines that need to be fed and several types of blanks that must be manipulated.

Our team placed a strong effort into reusing the standard KUKA platform as far as possible without the need of major alterations. However, it was clear from the beginning that a specific gripper was going to be needed. The solution for the showcase resulted from a trial and error approach emerging from a close cooperation between both partners (IMA and UAVR) that, through a few production iterations, converged to the final version of the gripper used for Showcase evaluation. The redesigned gripper includes only passive parts attached to the original gripper mounted in the KUKA platform.

\subsubsection{Pallet and Blank Perception}

Precise and robust pose estimation of blank is a necessary requirement to reliably pick a blank pile from tightly-aligned piles on a pallet (Fig. 7.12). In general all blanks in a pallet have the same shape and size. However, the printed patterns may vary. Therefore, the detection and pose estimations of blanks do not rely on conventional approaches such as color segmentation or local features matching such as SIFT [16]. To detect the blanks we propose a system composed of two modules: detection of a pallet and blank piles and pose estimation of a blank pile.

Since the exact location and configuration of the pallets are not known in advance, the mobile robot moves to a workbench in order to detect them. By using the depth information from the stereo camera installed on the pan-and-tilt unit, the detection of the pallets consists of three sequential steps: first, a voxel grid filter, based on the pallet's height is used to remove irrelevant points; second, the filtered points are projected on an horizontal plane and then a set of clusters is extracted to define candidate regions; and finally, the obtained clusters are compared against 2D template of a pallet projection to find its position and orientation. This same procedure is used to estimate of the piles positions by filtering the point cloud on the average height of the blank piles on the pallet.
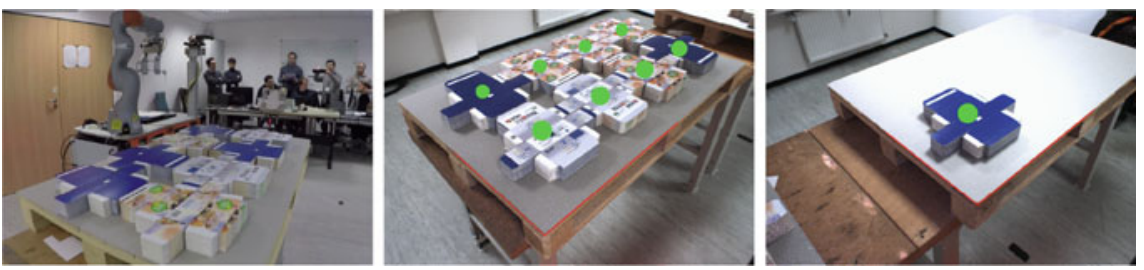

Fig. 7.12 Two pallets and blank piles and their detection results. The red lines in center and right images indicate the pose of pallets, while the green circles indicate the positions of blank piles 


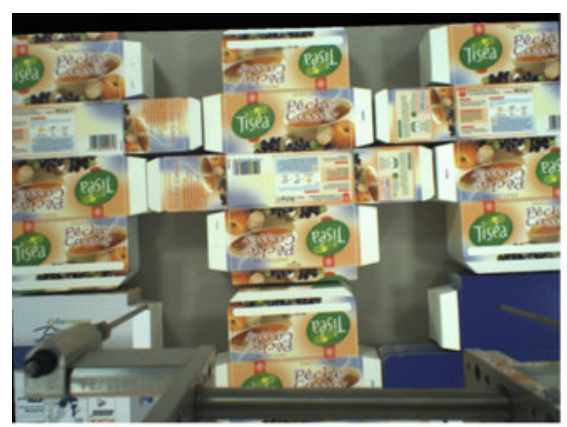

(a) original image

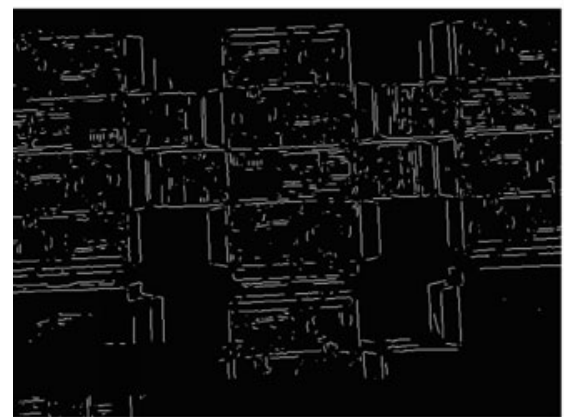

(c) outline detection with a pin filter

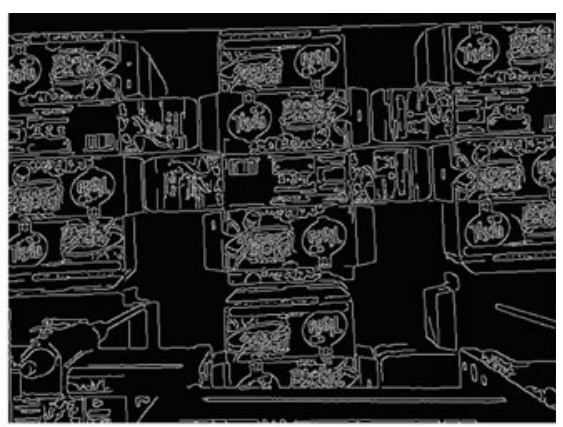

(b) edge detection with canny

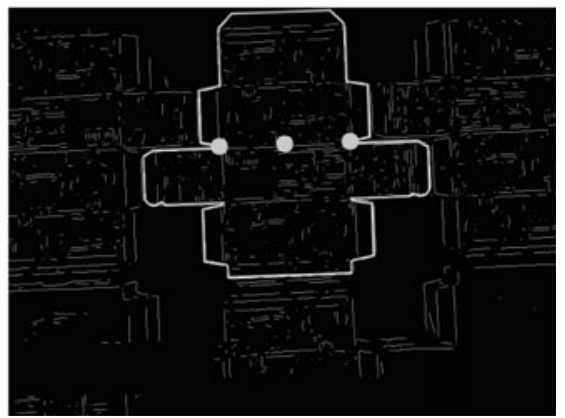

(d) blank template matching

Fig. 7.13 A sequential result of the pose estimation of a blank pile

Based on the initial estimated position of pallets and blank piles, the camera attached to the arm gripper is positioned to look vertically at the blank piles and capture an image (see Fig. 7.13a). Then, an edge detector (i.e. canny) is used to identify all edges in the images (see Fig. 7.13b). The resulting edges contain not only the edges from the blank outlines but also lines from inside the blank due to the printed patterns in the blanks. To reduce the edges that derive from the printing patterns, a pin filter is applied (see Fig. 7.13c). The kernel of the filter is defined as

$$
\begin{gathered}
K_{D P}=\left[x_{i}\right]= \begin{cases}\frac{p+h}{n}, & \text { if } x_{i} \text { is in the center } \\
\frac{h}{n}, & \text { otherwise }\end{cases} \\
\quad \begin{array}{c}
p>h \times|[x]|, \\
n=\sum x_{i},
\end{array}
\end{gathered}
$$

where $p$ and $h$ are the values of pin and head, respectively. The $p$ is larger than the sum of the heads and $n$ is the normalization factor. In the implementation the pin filter has a kernel of size $5 \times 5$ with $p=50$ and $h=1$. The reasoning is that the edges 
of outlines are straight lines and their points have a few number of neighbors while the printed patterns have many neighbors. Finally, to find the pose of the blank, the final filtered image is matched against a scaled template of the blank border, starting by checking the estimated position and then following a spiral path for the next test positions of the template until the threshold for blank detection are surpassed (see Fig. 7.13d).

\subsubsection{Motion Planning and Manipulation}

The blank pile to be manipulated is not a single rigid object, as shown in Fig. 7.14. It is a stack of card boards that not only bends under its own weight but blanks can easily break free from the pile due to the fact that blanks have low friction between each other. Any manipulation procedure of the a blank pile has to be aware of this fact. After picking, the pile has to be transported to the feeding magazine of the packaging machine. In the end, the blank pile has to be placed in the feeding magazine avoiding physical collision with the blanks that are already in place and with the four guiding rods that prevent blanks from slipping out of the inclined magazine surface.

By using a single arm with a gripper, the "picking" of a pile required, in the Showcase, three manipulation steps. Depending on the arrangement of the blank pile on the pallet, the robot needs to drag a blank pile over the edge of the pallet so there is enough free space for grasping without causing a breakdown or losing any blank. Then it has to grasp the pile from the edge and finally it has to recondition the pile for a firm grip. After grasping the blank pile, it is necessary to move the arm to the transport position, which is inside the mobile platform footprint, while having the arm in a pose that minimizes the necessary motions to place the blank pile in the feeding magazine. All manipulations have to be done while maintaining the blank pile in a pose that prevents it from breaking lose. This is a constraint that has to be accounted for when planning the motions of the robotic arm. To achieve the best motion trajectories that respected our constraint, several different motion planner were tested and benchmarked [17].

Due to the disposition of the blank piles on the pallet, certain piles are blocked by others and can not be manipulated. Therefore, the order in which the blank piles
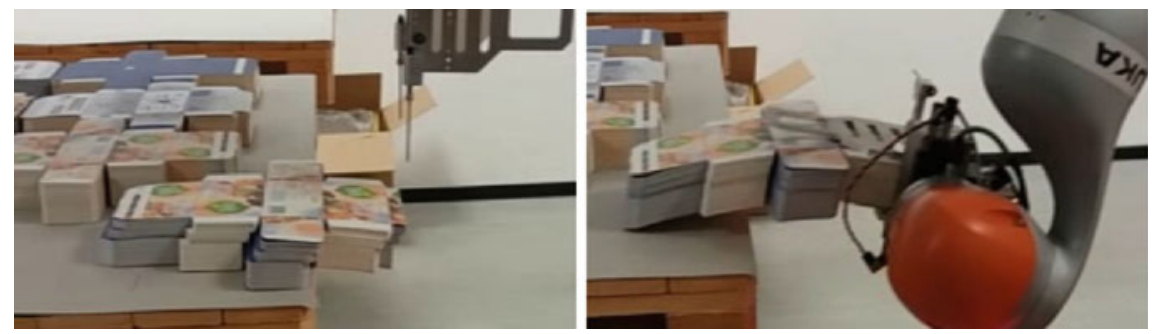

Fig. 7.14 Snapshots of bent blank piles 
are manipulated has to be planned. The planner considers the available blank piles on the available pallets and gives preference to the pallet with the lowest number of piles.

\subsubsection{Safe Human-Robot Collaboration}

Since industrial environments are noisy, verbal communication with human can be challenging and impractical with robots. Thus, gestures were considered as a practical alternative method of communication. The communication with the robot is therefore through gestures using the HRI system developed for the Freestyle use-case and reused for Showcase with changes only to interaction graph.

In the Showcase use-case, the human operator is no longer assumed to be confined to a single location. Now, the mobile platform and the operator can share the same physical workspace. In fact, it is expected that a human operator may physically approximate the robot in order to provide commands through gestures. This possible event raises safety concerns that result in an active and continuous monitorization of humans. The physical proximity of a human must result in a halt of moving parts of the mobile platform and, at the same time, it should position the relevant sensors in order to interpret possible order from the operator [18].

\subsubsection{Human Detection and Tracking}

The vision system integrated in the mobile platform (two stereo camera installed on the gripper and pan-and-tilt unit) have a limited field of view and shared access conflicts, i.e. if a camera is used for blank detection it can not be used for human detection. Therefore, to detect and track humans constantly, two laser range finders (LRF) were mounted on both sides of the mobile platform to cover all directions with a reduced area of shadow regions (i.e. areas that are no visible by the sensors). The multiple single-plane laser scans are merged to generate a virtual laser scan with a $360^{\circ}$ field of view.

The detection of a human is performed with a machine-learning-trained classifier that receives the virtual laser scan as input [18]. The positive and negative samples of laser scan readings of human body patterns were collected and trained with random forests beforehand [19]. In the tracking procedure, each detected human candidate in the virtual scan is matched, within a threshold, with the list of already tracked human. If it does not match, the candidate is added to the tracking list. Finally, all tracks are updated based on a Kalman filter.

The working environment of the Showcase adds an additional challenge to the human tracking system. The natural features of the environment, such as from a workbench, the robotic arm or blank piles, create false positives in the human tracking

module. To solve this issue, we introduced a region-based filtering and reasoning mechanism [18]. Most false positives arise from areas where the humans can not be physically, so those areas are marked as "black" rectangles and detection inside 
that areas are removed from the human detection. Additionally, a "green" rectangle defines the regions where human detection candidates are considered.

\subsubsection{Safety Management}

Safety is handled using the output of the human tracking module combined with a set of working regions, which are defined by the region-based filtering and reasoning mechanism. Two rectangular regions are defined around the mobile platform during navigation. An inner rectangle (a "red" rectangle) is close to the robot and an outer rectangle (a "yellow" rectangle) that covers a wider area. During arm manipulation, a circular region (a "red" circle) is defined around the arm, which radius is determined by the range of the arm. For the circular region, only the areas of the circle that do not intersect with the inner rectangle are considered.

Based on the fixed regions defined for human tracking and the dynamic regions around the mobile platform, the safety handle proceeds as follows. Any human candidate in the "green" region alone, is considered safe, i.e., the robot operation does not put any human in a "green" region in danger of physical harm. Therefore, no safety action is taken. If a human enters a "yellow" region, the navigation is suspended but the arm keeps doing its current operation. If a human enters a "red" region, then both the mobile platform and the arm are suspended until the human leaves the region in question. Both "yellow" and "red" regions also trigger HRI systems. HRI will take control of the pan-and-tilt unit to redirect the stereo camera to the human operator and awaits for a command every time "yellow" or "red" regions have humans. If the operator leaves the region of interest then the pan-and-tilt is released.

\subsubsection{Scenarios}

The Showcase use-case execution consisted of three different scenarios with increasing complexity. Here are their description:

Scenario 1 The goal of this challenge was to transport 2 blank piles to the feeding magazine. The robot had to identify the feeding magazine, the pallet and the piles.

Scenario 2 A second pallet is introduced to the task. The robot has to show that it selects the pallet with the lowest number of blanks first. Additionally, it has to switch pallets when needed and navigate accordingly.

Scenario 3 In this challenge the human operator was introduced. Now, the robot had to demonstrate safety features needed when humans share the robot environment complemented with human-robot interactions.

For the purpose of evaluation, several metrics had to be defined. Those include metrics for: perception, where the blanks the pallets and the feeding magazine had to be correctly detected; manipulation, where the manipulation of the blank piles had to be done correctly with safety concerns; and interaction, where the robot has to successfully track any possible human operator and be able to communicate. 


\subsubsection{Results}

The final outcome of the Showcase use-case was a scenario where a human operator and a mobile robot successfully work together to pick and place piles of blanks. All target metrics were reached with success which provided us the first place in the evaluation (out of five). The reusability of previously developed technologies is an important aspect for the success of our solution. This aspect facilitates integration of different technologies and facilitates the rapid development of new and also flexible solutions.

The Showcase evaluation was performed in an environment that, although not located in an industrial plant, includes the most important elements of the real environment where the task will be executed as a final product. It uses real pallets with real piles of blanks and a prototype of the blank feeding mechanism of a packaging machine that includes all relevant features for the task to be performed. In the industrial setting, the distance between the blank feeding mechanism and the pallets may be larger, there may be several packaging machines that need to be fed and several types of blanks that must be manipulated.

\subsubsection{Field Tests}

The Field Tests use-case is an evolution of the Showcase that takes us closer to Autonomous Blank Feeding for Packaging Machines in a industrial environment (Fig. 7.15). TIMAIRIS field tests use-case was composed of a shop floor version of the complete blank feeding problem. It included: two packaging machines using different blanks and with different feeder mechanisms at their blank magazines; the mechanism that feeds the blanks into the packaging machine; and three pallets filled in with $15 \mathrm{~cm}$ height blank piles used in production. The task consisted in recognize the blank piles on the three pallets, devise a plan for picking them considering the packaging machines blank consumption rates and current blank level at each magazine, and finally transport and place them in the blank magazines.

\subsubsection{General Architecture}

The Field Tests use-case introduced the online monitoring of the blank feeding level, which is provided by external components to the mobile platform. Also, there is an external dashboard that provides relevant information to the operator. The introduction of these external components expanded our architecture, centered in the mobile platform, to a factory-wise architecture. The architecture is depicted in Fig. 7.16.

For the Field Tests the robot platform was enhanced to include a new gripper device that includes a parallel 2-jaw gripper from Zimmer with a higher gripping force that enables manipulation of $15 \mathrm{~cm}$ high piles. The new gripper mechanical fingers allow picking the blank pile from the pallet in the vertical direction. Another 


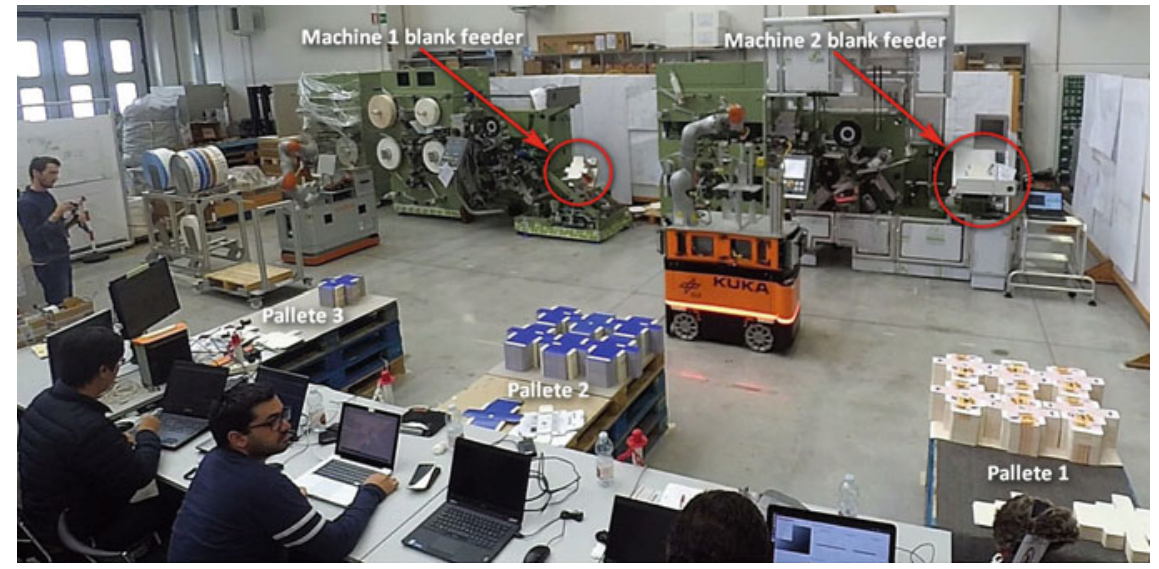

Fig. 7.15 Field Tests environment layout. It includes three pallets and 2 machines with blank feeders

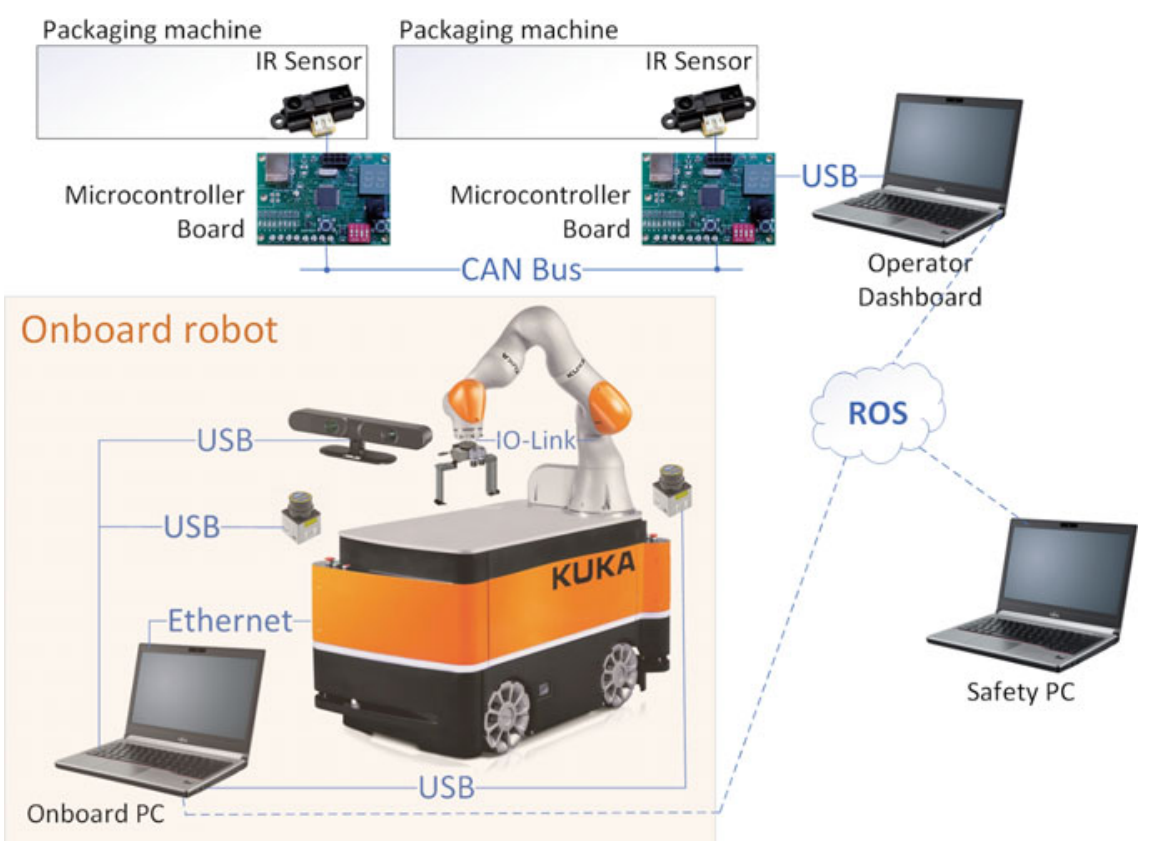

Fig. 7.16 TIMAIRIS architecture used in the field tests use-case 
enhancement was the inclusion in the robot platform of a pile buffer that can be used to store a pile, while the other is being transported by the gripper, and a rotation buffer, used to re-grasp the piles in order to be able to place them at the feeders.

The LRFs used for human tracking in the Showcase have also been used in the Field Tests, as well as the Asus Xtion, that has been used mainly for human-robot interaction. An onboard PC runs the solver and is connected to ROS communication network.

IR distance sensors have been added to the feeders of both packaging machines to measure the blank level. These sensors are managed by a microcontroller board and connect to the operator dashboard (a laptop PC running ROS) using a CAN bus. This architecture is prepared for the expansion of the number of packaging machines. The safety PC, that runs the safety approach and human tracking procedures, is an external laptop, that communicates with others through ROS.

\subsubsection{Perception}

Field Tests perception was strongly based on the algorithms developed for the Showcase. The main changes are in the improvement of some implementations, that allowed blank detection, for example, to become 10x faster, and in the selection of the observation points for pallet and magazine detection that had to be adapted to the new environment (Fig. 7.17a).

By changing the blank detection algorithm, using the same principles, from counting the number matching pixels for each border template position and rotation, to an approach where this computation is performed using a convolution filter and the maximum of the filter result defines the best pose, the speed of this detection has been increased by 10x, without changing the final results (Fig. 7.17b).

Near each blank magazine, an ARUCO marker has been added to measure the displacement from the robot to the magazine (Fig. 7.17c). This displacement is critical because the manipulation to place the blank pile must be very precise to be successful. ARUCO markers have been placed in a position that is mostly horizontal, to increase the sensitivity of their localization in the most critical angles. The procedure for using markers to perform this correction has been generalized.

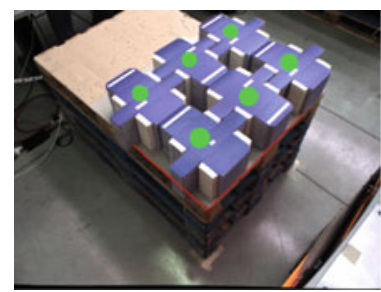

(a) pallet and pile detection

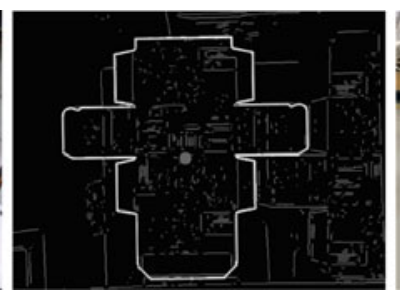

(b) blank detection

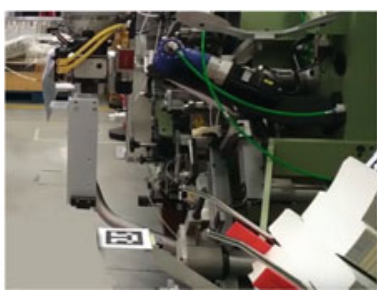

(c) marker detection

Fig. 7.17 Examples of perception in the Field Tests use-case 


\subsubsection{Planning}

In the Field Tests use-case, the robot had to feed blanks at 2 packaging machines. In addition, the blanks shapes for these 2 machines were different and the blank magazines had different capacities and restrictions for blank loading. In order to get a general solution which can be adapted to the number of packaging machines and to their characteristics, the sequence of operations was derived using a PDDL 2.1 planner (named popf) [20] for high-level actions and a low-level planner for choosing the picking blank pile within the pallets. The high-level actions that were considered were the following:

- move ?from ?to

- pick ?pile ?waypoint ?gripper

- pick-to-buffer ?pile ?waypoint ?gripper

- place-big ?pile ?waypoint ?gripper

- place-small ?pile ?waypoint ?gripper

- place-from-buffer ?pile ?waypoint ?gripper

- human-disarm ?pile

where big and small designate the 2 types of blanks that were used in the Field Tests. The parameters of the planning problem are:

- number of packaging machines

- type of blanks for each packaging machine

- initial position of robot and available blank piles

- initial blank level at each blank magazine

- rate of consumption of blanks for each packaging machine

- highest and lowest blank levels that allow inserting a new pile in each magazine

- estimated duration of each high level operation.

The problem of planning is rather complex as the environment is not stationary. The packaging machines are always working, decreasing the blank level at their magazines, while the robot is in operation. The plan should make sure that all machines are fed with blanks, considering the restrictions of the blank magazines, without any of them getting at the lowest level, to ensure continuous operation.

The domain of this problem was modelled using PDDL 2.1 with durative-actions. To model the non-stationary part of the environment, PDDL functions for the magazine level of each magazine were used. The value of these functions is updated for every action, according to the action duration. Some step actions had to be inserted to model periods where the robot must wait without performing any of the high-level actions. These step actions keep the magazine levels updated.

The planning problem is implemented by a ROS node that implements the service get_plan. This service reads the magazine levels, detected by the IR sensors at each magazine, considers the blank piles of each type present at the pallets and generates the PDDL problem description. Planning is performed using the popf planner from the ROSPlan framework [21]. The service continues to parse the plan generated by 


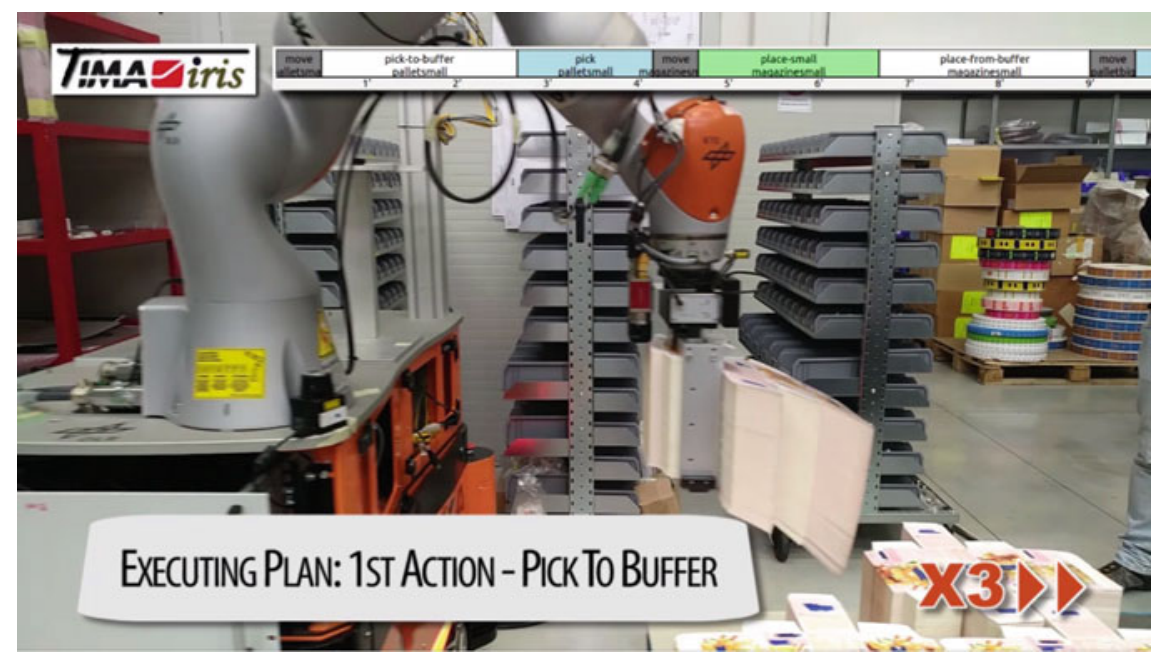

Fig. 7.18 Snapshot of the the robot following the first step of the execution plan

popf, publishes the plan and makes it part of the service response. Figure 7.18 presents the robot executing the first action of the plan that is shown in the top of the figure. A graphical user interface has also been developed that allows continuous monitoring of the blank levels, a visualization of the current plan, and an offline planning mode where the plan parameters can be edited interactively, instead of being read from the environment, and that was used for testing the planner (Fig. 7.19).

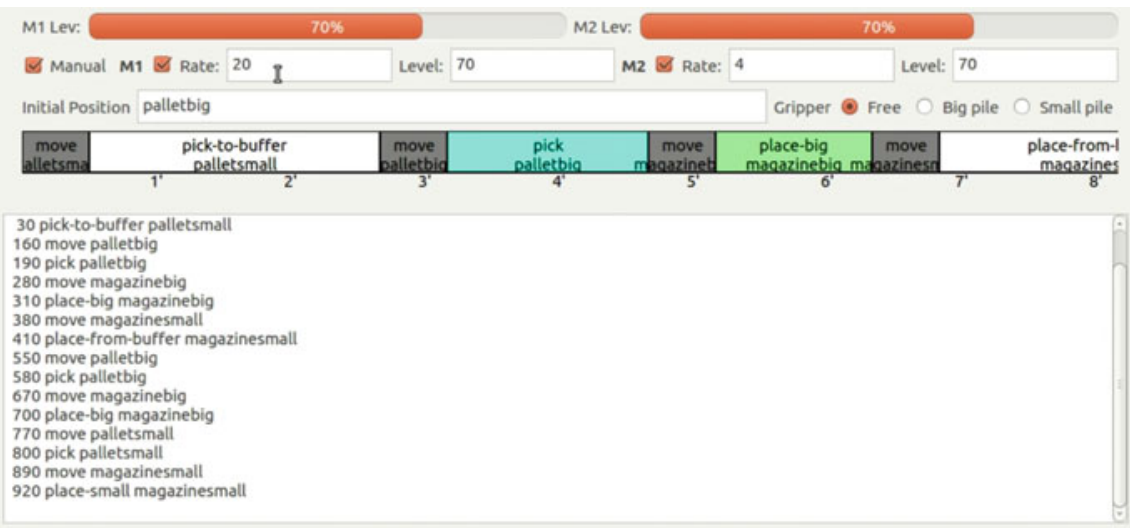

Fig. 7.19 Execution dashboard. Its show the state of the feeding magazine level, the current plan and an execution log. It also allows to set several execution parameters 


\subsubsection{Manipulation and Safety/Interaction}

Field Tests manipulation had to be re-engineered from the approach used in the Showcase due to the different gripper. Additional changes to manipulation are the actions performed by the robot arm to put the pile in the rotation buffer and pick it again, as well as the placing of the pile in the transport buffer. Nonetheless, most manipulation actions are similar to the ones performed in the Showcase, thus reducing the development time. Example of manipulation action performed in the Field Tests use-case are shown in Fig. 7.20.

The safety/interaction part of the Field Tests use-case is the same from the Showcase. The difference is that now multiple humans can be present in the environment. Now the safety management assumes that the human operator is the one closest to the pan-and-tilt unit. An example of interaction and human tracking is shown in Fig. 7.21.

\subsubsection{Scenarios}

TIMAIRIS Field Tests evaluation was structured around 3 different scenarios of increasing complexity. These scenarios play a similar role as the challenges used for the freestyle and showcase use-cases.

(a)

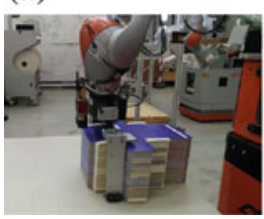

(b)

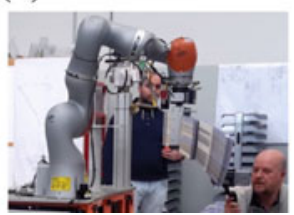

(c)

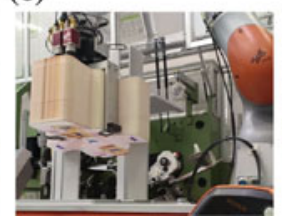

(d)

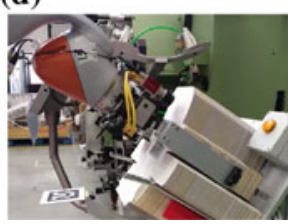

Fig. 7.20 Example of manipulation actions used in the Field Tests use-case. a Picking with compliance. b Picking of non-rigid objects. $\mathbf{c}$ Using the pile buffer. d Smooth placing in the feeding magazine

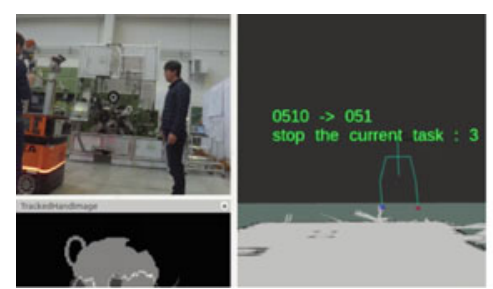

(a) gesture recognition

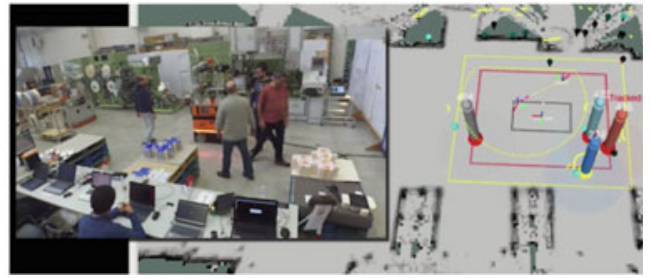

(b) human tracking and safety regions

Fig. 7.21 Example of human-robot interaction and human tracking 


\subsubsection{Results}

The Field Tests have demonstrated the feasibility of practical use of an intelligent mobile robot in this scenario, by exploiting the following technologies: skill-based anytime agent architecture, in which the agent can start execution at any task state; knowledge-based perception, that enables the robot to both quickly detect the pose of the pallets and blank magazines using spatial knowledge, and to recognize the pose of densely and closely lying blank piles; a set of skills and a sophisticated gripper which enable the robot to grasp and adjust an untied pile of non rigid blanks, move the arm into a safe navigation posture and to smoothly feed the pile to two different complex shaped blank magazines; planning and re-planning, which produces a sequence of actions for manipulation; rich multimodal interaction which enables the robot to interact with human collaborators in a noisy environment by encoding a set of commands with a fixed number of symbols.

The Field Tests workspace mimics the conditions that will be found in a real scenario. Efficient navigation on reduced spaces, two complete packaging machines with two different blank feeding devices, including the physical limitations imposed by the mechanical cluttering, non deterministic location of the blank pallets, changing illumination conditions and the presence and interaction with human co-workers. The excellent results obtained during the field tests phase, which did not focus on speed efficiency, can in fact demonstrate in a clear and provable way that the final prototype will be able to work under realistic conditions. Note also that the typical feeding rate of a packaging machine is around $1 \mathrm{~cm} / \mathrm{min}$ for large blanks and $0.8 \mathrm{~cm} / \mathrm{s}$ for small blanks. In this field test, the robot was able to feed a $15 \mathrm{~cm}$ pile to the feeder within a maximum time of $2 \mathrm{~min}$. Therefore, with the current solution, not optimized for speed, the robot would already be able to feed four packaging machines, which confirms the results provided by the planner.

\subsection{Conclusion}

IMA's position of world leadership in the production of packaging machines requires a constant focus on staying ahead of the curve in terms of technological innovation. The industrial world is looking more and more at automation as a resource to manufacture better products in a cost-efficient way, and this area is of utmost importance for IMA, too. EuRoC is not the only IMA's R\&D project on robotics and automation; in fact, automation is one of the two/three most important areas the IMA research staff is involved in.

The current results of the EuRoC project are in line with those that were stated at the project submission. The convenience of robotised features reflects into customers', workers', and sustainability benefits. From the customers' perspective, robotised systems would make it possible to avoid the use of a human operator dedicated to blank loading, resulting into cost cutting and efficiency gaining. This also would give the operator more time for other operations such as quality control, or looking 
after more machines. From the workers' point of view, the adoption of robots to automate repetitive procedures is more than welcome. Such robotised systems alleviate the stress derived from repetitive labour and contribute to build real factories for humans.

As for sustainability, adopting robotised systems also affects the sustainability of the entire packaging process. In fact, fewer mistakes in feeding wrong blanks, or feeding them in the wrong way, results into less waste, fewer machine stoppages, and a reduced energy consumption - a mandatory requirement for a green factory.

TIMAIRIS is confident that the overall results of the project-and of IMA's commitment to robotics and automation-will manifest themselves in a stronger leadership position, harder and harder to overcome, and in a wider presence on the international markets. Moreover, the EuRoC project has given IMA the opportunity to develop links with research centers with which to find innovative solutions to automation issues. This type of knowledge will be deepened and used to find innovative solutions to many other problems. IMA and UAVR signed a contract, funded by IMA, to continue their collaboration during 2018/2019.

It is difficult to imagine the future of industry and manufacturing without a massive role being played by robotics and automation. IMA is actively working on this topic, both in the framework of the EuRoC project and in other R\&D projects. The results of this wide range of activities will constitute the backbone of a new way of designing machines that employ robots and automation in general, the future IMA's products will have to be designed around the notion that an increasing number of repetitive and low-skilled actions will be carried out by machines.

The EuRoC project will bring IMA to develop new products that support and complement its automatic machines, as well as new services associated to cooperative robotised platforms. Also to be stressed is that the use of this kind of robotised blank feeding is intended to be included as an option in future packaging machine products as soon as its robustness reaches the required Technology Readiness Level.

Overall, the EuRoC project had a strong impact both on UAVR and IMA. EuRoC provided the framework to start the collaboration between these 2 institutions and this collaboration is active and effective after EuRoC is finished. The complementarity of UAVR and IMA is well represented in Fig. 7.22.

Some of the strong points in the EuRoC project, from the TIMAIRIS perspective, are its mandatory cooperation between academia and industry focused on specific challenging applications; the structure of EuRoC competition (Stages I, II and III), their respective tasks, the rank based filtering of teams for entering new stages and the provided funding worked very well to foster a productive environment. The interaction between TIMAIRIS and Challenge 2 host, and the tools, including ROS wrapper, eased-up the developments and the exchange of ideas and feedback from host was always very important; the meetings and workshops that joined all EuRoC teams, core partners and evaluators was very enriching.

Some points that, from TIMAIRIS perspective, could be improved are: some of the evaluation models should be thought more carefully, as for example allowing some metrics to dominate the evaluation in Stage II; midterm evaluation workshop format was not clear (great improvement was performed for Showcase and Field Tests 


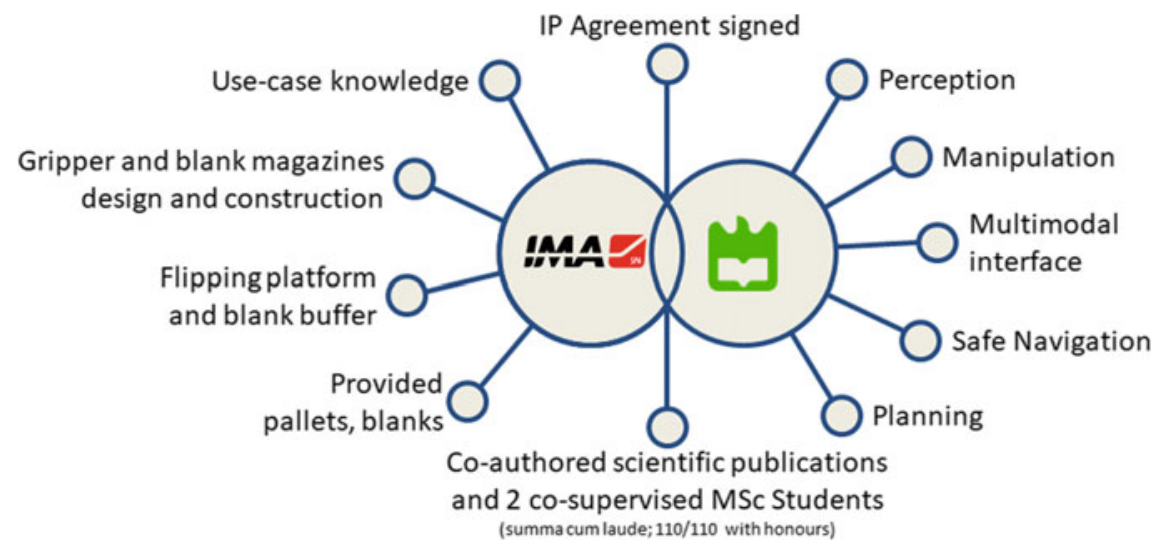

Fig. 7.22 Complementarity between UAVR and IMA

workshops); some deadline dates and penalties for missing them were not clear or conveyed to the teams with a short notice.

TIMAIRIS would especially like to acknowledge the specification of the succession of Benchmarking and Freestyle (Stage IIa); Showcase (Stage IIb) and Field Tests (Stage III) and their competitive/selective nature that provided a very good ramp up for the development towards a successful solution for the industrial problem to be solved. The Benchmarking was a very important tool to get to know the EuRoC Challenge 2 robot platform capabilities, and also its limitations; increase the mutual knowledge and trust between UAVR and IMA and also between these 2 institutions and Challenge 2 host (DLR). TIMAIRIS Freestyle specification was provided by TIMAIRIS in its Stage II proposal. This specification was not directly related to IMA's industrial problem, but it provided for the development of many technologies that were used in the next stages. The freestyle objectives were placed at a very high level, and were very well demonstrated during the evaluation. The Showcase provided for a first implementation of autonomous blank feeding in a lab setup. Finally, the Field Tests allowed the development of solutions in an industrial environment, very close to the final product.

We would like to thank the EuRoC Core partners, and also Challenge 2 host, for their effort in the EuRoC specification and management and for providing the framework for a very productive TIMAIRIS research and development work.

Acknowledgements This work was supported by the EuRoC Project under Grant no. 608849 and by Portuguese National Funds through the FCT-Foundation for Science and Technology, in the context of the project UID/CEC/00127/2019. 


\section{References}

1. Hermann, M., Pentek, T., Otto, B.: Design principles for industrie 4.0 scenarios. In: 201649 th Hawaii International Conference on System Sciences (HICSS), pp. 3928-3937. IEEE (2016)

2. Bischoff, R., Guhl, T.: The strategic research agenda for robotics in Rurope [industrial activities]. IEEE Robot. Autom. Mag. 17(1), 15-16 (2010)

3. Siciliano, B., Caccavale, F., Zwicker, E., Achtelik, M., Mansard, N., Borst, C., Achtelik, M., Jepsen, N.O., Awad, R., Bischoff, R.: Euroc - the challenge initiative for European robotics. In: ISR/Robotik 2014; 41st International Symposium on Robotics, pp. 1-7. VDE (2014)

4. Pedrocchi, N., Vicentini, F., Matteo, M., Tosatti, L.M.: Safe human-robot cooperation in an industrial environment. Int. J. Adv. Robot. Syst. 10(1), 27 (2013)

5. Quigley, M., Conley, K., Gerkey, B., Faust, J., Foote, T., Leibs, J., Wheeler, R., Ng, A.Y.: ROS: an open-source robot operating system. In: ICRA Workshop on Open Source Software, vol. 3, p. 5. Kobe, Japan (2009)

6. Pedrosa, E., Lau, N., Pereira, A., Cunha, B.: A skill-based architecture for pick and place manipulation tasks. In: Pereira, F., Machado, P., Costa, E., Cardoso, A. (eds.) Progress in Artificial Intelligence, pp. 457-468, Coimbra, Portugal, September 2015. Springer International Publishing

7. Amaral, F., Pedrosa, E., Lim, G.H., Shafii, N., Pereira, A., Azevedo, J.L., Cunha, B., Reis, L.P., Badini, S., Lau, N.: Skill-based anytime agent architecture for logistics and manipulation tasks: EuRoC Challenge 2, Stage II-Realistic Labs: Benchmarking. In: 2017 IEEE International Conference on Autonomous Robot Systems and Competitions, ICARSC 2017, pp. 198-203 (2017)

8. Rusu, R.B., Marton, Z.C., Blodow, N., Dolha, M., Beetz, M.: Towards 3D point cloud based object maps for household environments. Robot. Auton. Syst. 56(11), 927-941 (2008). Semantic Knowledge in Robotics

9. Rusu, R.B.: Semantic 3D object maps for everyday manipulation in human living environments. Ph.D. Thesis, Computer Science department, Technische Universitaet Muenchen, Germany, October 2009

10. Hamidreza Kasaei, S., Seabra Lopes, L., Tomé, A.M., Oliveira, M.: An orthographic descriptor for 3D object learning and recognition. In: 2016 IEEE/RSJ International Conference on Intelligent Robots and Systems (IROS), pp. 4158-4163 (2016)

11. Pedrosa, E., Pereira, A., Lau, N.: A scan matching approach to SLAM with a dynamic likelihood field. In: 2016 International Conference on Autonomous Robot Systems and Competitions (ICARSC), pp. 35-40, Portugal, Bragança. IEEE (2016)

12. Lim, G.H., Pedrosa, E., Amaral, F., Lau, N., Pereira, A., Dias, P., Azevedo, J.L., Cunha, B., Reis, L.P.: Rich and robust human-robot interaction on gesture recognition for assembly tasks. In: 2017 IEEE International Conference on Autonomous Robot Systems and Competitions, ICARSC 2017, pp. 159-164 (2017)

13. Gleeson, B., MacLean, K., Haddadi, A., Croft, E., Alcazar, J.: Gestures for industry: intuitive human-robot communication from human observation. In: Proceedings of the 8th ACM/IEEE International Conference on Human-Robot Interaction, pp. 349-356. IEEE Press (2013)

14. Lim, G.H., Suh, I.H.: Robust robot knowledge instantiation for intelligent service robots. Intell. Serv. Robot. 3(2), 115-123 (2010)

15. Norvig, P.: Natural language corpus data. In: Beautiful Data, pp. 219-242 (2009)

16. Lowe, D.G.: Object recognition from local scale-invariant features. In: The Proceedings of the Seventh IEEE International Conference on Computer Vision, vol. 2, pp. 1150-1157. IEEE (1999)

17. Tudico, A., Lau, N., Pedrosa, E., Amaral, F., Mazzotti, C., Carricato, M.: Improving and benchmarking motion planning for a mobile manipulator operating in unstructured environments. In: Portuguese Conference on Artificial Intelligence, pp. 498-509. Springer (2017)

18. Lim, G.H., Pedrosa, E., Amaral, F., Dias, R., Pereira, A., Lau, N., Azevedo, J., Cunha, B., Reis, L.P.: Human-robot collaboration and safety management for logistics and manipulation tasks. 
In: Ollero, A., Sanfeliu, A., Montano, L., Lau, N., Cardeira, C. (eds.) Advances in Intelligent Systems and Computing, vol. 694, pp. 15-27. Portugal. Springer International Publishing, Porto (2017)

19. Breiman, L.: Random forests. Mach. Learn. 45(1), 5-32 (2001)

20. Coles, A., Coles, A., Fox, M., Long, D.: Forward-chaining partial-order planning. In: Proceedings of the Twentieth International Conference on International Conference on Automated Planning and Scheduling, ICAPS'10, pp. 42-49. AAAI Press (2010)

21. Cashmore, M., Fox, M., Long, D., Magazzeni, D., Ridder, B., Carreraa, A., Palomeras, N., Hurtós, N., Carrerasa, M.: ROSPlan: planning in the robot operating system. In: Proceedings of the Twenty-Fifth International Conference on International Conference on Automated Planning and Scheduling, ICAPS'15, pp. 333-341. AAAI Press (2015) 